Pensamiento Crítico Vol.17. N¹, pp. 19-30

\title{
Estructura y tipos de créditos de la Banca Múltiple en el Perú
}

\author{
Mg. Gaby Cortez Cortez
}

\section{RESUMEN}

La estructura y tipos de crédito de la Banca Múltiple en el Perú es el resultado de las estrategias y de la posición dominante que tienen algunos bancos del sistema para diversificar sus operaciones financieras, ofreciendo servicios a clientes con fuerte presencia en los diversos sectores de la economía; dejando espacios reducidos al resto de bancos que cuentan con menor poder económico y capacidad operativa para el desarrollo de sus actividades.

Palabras clave: Crédito, margen financiero, ROE.

\begin{abstract}
The structure and types of credit of Commercial Banks in Peru, is the result of the strategies and the dominant position held by some banks in the system to diversify its financial operations, and serve customers with a strong presence in various sectors of the economy, leaving spaces to other banks that have less economic power and operational capability for the development of its activities.
\end{abstract}

Key words: credit, financial spread, ROE. 


\section{Pensamiento Crítico Vol.I7. No I}

\section{Introducción}

El mercado bancario en el Perú está conformado por un conjunto de bancos que se diferencian entre sí por su capacidad de operación, la magnitud de sus activos, así como por los depósitos captados. Esta diferencia determina una estructura en los tipos de créditos y tasas de interés, conforme a las estrategias aplicadas por los bancos respecto a sus clientes, a la ubicación en ciertos sectores económicos y a la localización en áreas geográficas específicas del territorio nacional.

\section{Gestión de la Banca Múltiple}

\section{Activos y pasivos}

Los activos de la Banca Múltiple en su conjunto al 31 de diciembre de 2011 ascendieron a S/. 190,710 millones de nuevos soles y se captaron depósitos por un monto de S/. 171,254 millones de nuevos soles. Los Bancos de Crédito y Continental en el mismo año tenían en conjunto activo por un monto de S/.109,723 millones de nuevos soles, representando en términos porcentuales $57.53 \%$ del total de activos del sistema. Por el lado del pasivo, ambos bancos captaron depósitos por un monto de S/. 99,712 millones de nuevos soles, representando el 58,22\% de los depósitos totales. Igualmente, los bancos Scotiabank e Interbank mostraron en conjunto activos por un monto S/.48,752 millones de nuevos soles y pasivos ascendentes a S/. 42,777 millones de nuevos soles, con porcentajes respecto a los totales del sistema de $25.56 \%$ y $24,98 \%$ respectivamente. Finalmente, el resto de bancos que conforman el sistema en el Perú tenían activos por $\mathrm{S} / .21,618$ millones de nuevos soles (16.9\%) y pasivos por $\mathrm{S} / .20,456$ millones de soles (16.8\%).

Con el objeto de agrupar al conjunto de la Banca Múltiple en el Perú según activos y pasivos, se ha elaborado una estructura en la cual coexisten bancos a los cuales denominaremos Bancos Grandes: Bancos de Crédito y Continental; Bancos Intermedios: Bancos Scotiabank e Interbank; Bancos Medianos: Financiero, Interamericano de Finanzas, y Mibanco; Bancos Pequeños: De Comercio, Falabella, Ripley y Azteca ; y otros bancos agrupados bajo una tipología diferente: Citibank, Santander, HSBC y Deutsche Bank que actúan en áreas muy especificas y los hemos denominado como Bancos Sucursales. 


\section{Gaby Cortez Cortez}

\section{Sectores económicos}

Una política aplicada por casi la totalidad de los bancos en el Perú, es la de diversificar sus créditos en el mayor número de sectores económicos, con excepción de los bancos generalmente pequeños, los cuales centralizan sus operaciones en créditos para el consumo. La Superintendencia de Banca y Seguros presenta estadísticas de créditos por sectores económicos y empresa bancaria en las que se muestran 16 de estos sectores.

Al 31 de diciembre de 2011, el Banco de Crédito lidera las colocaciones con una presencia en 9 sectores económicos, y ocupa el segundo lugar en 6 sectores. El Banco Continental ocupa el primer lugar en 4 sectores, y el segundo lugar en 7 sectores. El Scotiabank ocupa el primer lugar en solo 2 sectores y el segundo lugar en 3 sectores; sin embargo, ocupa el tercer lugar, siguiendo al Banco de Crédito y Continental en 9 sectores. Finalmente, el Banco Interbank ocupa el tercer lugar en un solo sector económico (Agricultura, Ganadería, Caza y Silvicultura). El restante conjunto de bancos del sistema no tienen presencia importante en algún sector de la economía.

De la totalidad de recursos destinados por la Banca Múltiple a los sectores productores de bienes y servicios el Banco de Crédito participa con el $35.98 \%$ de la totalidad de recursos; el Banco Continental destina el 25.14\%; el Scotiabank el 16.19\%; el Interbank el $8.29 \%$, y el resto de los bancos en conjunto destinan el $14.4 \%$.

El grado de atención a los sectores económicos por cada uno de los bancos es distinto, y corresponde a las estrategias que tienen los bancos según su posibilidad de dominio en cada uno de estos sectores. Hay sectores que son claves en la producción de bienes y servicios, como es el caso del sector industria manufacturera, comercio, construcción, seguido en menor medida del sector minero (en el cual el Banco de Crédito tiene una presencia importante) y el sector agricultura, ganadería, caza y silvicultura.

A continuación, se muestra la participación en porcentaje de las colocaciones de los bancos más importantes en cinco sectores considerados claves de la economía peruana: 1) industria manufacturera; 2) comercio; 3) construcción; 4) minería y 5) agricultura, ganadería, caza y silvicultura, al 31 de diciembre de 2011: 


\section{Pensamiento Crítico Vol.I7. No I}

La participación de recursos colocados por los bancos en el sector industria manufacturera fue el siguiente:

$\begin{array}{lr}\text { Crédito } & 61.07 \% \\ \text { Continental } & 22.93 \% \\ \text { Scotiabank } & 6.27 \% \\ \text { Interbank } & 5.26 \%\end{array}$

La participación de recursos colocados en el sector comercio fue:

Crédito

Continental

Scotiabank

Interbank
$49.45 \%$

$24.74 \%$

$9.36 \%$

$8.23 \%$

La participación de recursos colocados en el sector construcción es como sigue:

Crédito

Continental

Interbank

Scotiabank
$54.89 \%$

$26.99 \%$

$5.99 \%$

$4.41 \%$

La participación porcentual de recursos colocados en el sector minería:

Crédito

Continental

Scotiabank

Interbank
$80.42 \%$

$9.91 \%$

$4.18 \%$

$3.65 \%$

La participación porcentual de las colocaciones en el sector agricultura, ganadería. caza y silvicultura: 


\section{Gaby Cortez Cortez}

$\begin{array}{ll}\text { Crédito } & 40.96 \% \\ \text { Continental } & 27.54 \% \\ \text { Interbank } & 13.84 \% \\ \text { Scotiabank } & 13.70 \%\end{array}$

La participación en el total de recursos colocados en estos sectores se indica a continuación:

$\begin{array}{lr}\text { Banco de Crédito } & 49.87 \% \\ \text { Continental } & 29.25 \% \\ \text { Scotiabank } & 7.56 \% \\ \text { Interbank } & 4.98 \%\end{array}$

Estas estadísticas nos muestran una estructura de créditos que concentra en dos bancos (Crédito y Continental) una parte importante de las colocaciones en sectores claves de la economía peruana; seguida por una posición intermedia de otros dos bancos (Scotiabank e Interbank). En su conjunto estos cuatro bancos concentran el 91.66\% de sus colocaciones en estos sectores seleccionados. Estas cifras son bastante significativas por cuanto nos muestran un mercado de crédito bastante concentrado, dejando espacios pequeños para el desarrollo de los bancos restantes del sistema, los cuales se ubican básicamente, en créditos a la pequeña y micro empresa (Mibanco), y créditos de consumo.

\section{Créditos por tipo de empresa}

El tipo de cliente y su importancia en el mercado marca la pauta en los créditos de los bancos del sistema. Los bancos a los que se ha denominado como Bancos Grandes (Crédito y Continental) y los Bancos Intermedios (Scotiabank e Interbank) dirigen sus créditos a las empresas corporativas, grandes y medianas. Los Bancos Medianos dirigen sus créditos a las empresas medianas, pequeñas y microempresas. Los Bancos Pequeños direccionan principalmente sus créditos para el consumo. Los Bancos Sucursales, dirigen sus recursos en menor escala que los bancos grandes hacia los sectores corporativos y de grandes empresas, en operaciones de descuento, préstamos y arrendamiento financiero. 


\section{Pensamiento Crítico Vol.I7. No I}

Esta clasificación tiene sus matices debido a que los bancos pueden colocar recursos en algunos nichos cuando estos se encuentran maduros o adquieren cierta importancia, como es el caso de los créditos a la pequeña y microempresa, consumo o hipotecario. El ingreso de los bancos considerados grandes o intermedios en estos tipos de crédito pueden comprometer el desarrollo de los bancos medianos y pequeños, los que debido a esta situación deben reconsiderar sus estrategias de permanencia en el mercado.

La superintendencia de banca y seguros muestra estadísticas de crédito a las empresas bajo la denominación de: 1) Créditos Corporativos, 2) a Grandes Empresas, 3) a Medianas Empresas, 4) a Pequeñas Empresas, 5) a Microempresas, 6) de Consumo y 7) Créditos hipotecarios para vivienda. Bajo este criterio, al 31 de diciembre de 2011, el Banco de Crédito muestra liderazgo en las dos primeras líneas (Corporativo y a Grandes Empresas) con el $42.62 \%$ y $39.53 \%$ respectivamente. El Banco Continental domina los créditos para la Mediana Empresa con un 34.33\%, seguido por el Banco de Crédito con el $27.32 \%$.

El Banco de Crédito tiene supremacía en 5 de los 7 tipos de crédito dirigidos a las empresas. Mibanco logra posicionarse en el primer lugar de los créditos a las microempresas con $47.06 \%$ del total de colocaciones en este rubro, seguido por el Banco de Crédito con un $21.68 \%$. Aquí se puede notar cómo sectores que no eran atendidos prioritariamente por los bancos denominados grandes, ahora adquieren cierta atención por estos.

En el caso de los créditos de consumo, los bancos pequeños enfrentan una fuerte competencia por la participación de los Bancos de Crédito y Scotiabank con el 34.58\% y el $13.93 \%$ correspondientes; mientras que, las colocaciones de los bancos Falabella y Ripley fueron de 7.89 y $4.52 \%$, respectivamente.

\section{Tasa de interés}

\section{Tasa de interés activa}

Las tasas de interés que cobran los bancos, y las que pagan por los depósitos, no solo se aplican en teoría por la interacción de la oferta y la demanda, sino además, por 


\section{Gaby Cortez Cortez}

la necesidad básica de encontrar un margen que permita a estos bancos obtener una tasa de retorno del capital esperada, aún en situaciones de competencia imperfecta.

Si bien, en términos generales, los bancos buscan una presencia importante en el mayor número de sectores y espacios económicos, al interior de estos sectores y espacios hay una distribución de sus colocaciones conforme a la capacidad y política crediticia de los mismos. Los bancos a los cuales se les ha denominado "grandes" dirigen sus créditos con tasas menores aplicadas a las empresas corporativas o grandes empresas. Por una suerte de repartición implícita, los bancos medianos dirigen sus créditos, principalmente a las empresas medianas con tasas de interés algo más altas que las tasas que se cobran a las empresas corporativas y grandes empresas. Estas tasas se van elevando conforme los clientes o empresas tengan una menor capacidad económica, tal es así que a las empresas pequeñas y microempresas se les cobra tasas de interés mucho más altas que las que se aplican a las empresas de los escalones superiores (corporativas, grandes y medianas). Las tasas de interés para créditos de consumo también son tasas altas si las comparamos con las tasas aplicadas a las empresas corporativas, grandes y medianas.

A continuación, se muestra en el Cuadro 1 la tasa de interés activa promedio en moneda nacional por tipo de empresas y, en el Cuadro 2, las tasas de interés activas promedio en moneda extranjera por tipo de empresas, según la nomenclatura aplicada por la SBS:

Cuadro No. 1

Tasas de Interés Activas Anuales de las Operaciones en Moneda Nacional de los Bancos

(Realizadas en los últimos 30 días por tipo de crédito al 29/12/2011)

\begin{tabular}{lcccccc}
\hline & \multicolumn{5}{c}{ Bancos } & \\
\cline { 2 - 6 } Empresas & Grandes & Intermedios & Medianos & Pequeños & Sucursales & Promedio \\
\hline Corporativos & 5.8 & 5.8 & 6.3 & & 7.0 & 6.2 \\
Grandes Empresas & 7.3 & 6.8 & 7.9 & 11.8 & 7.7 & 8.3 \\
Medianas Empresas & 11.5 & 10.7 & 9.7 & 15.8 & 7.9 & 11.1 \\
Pequeñas Empresas & 18.9 & 22.4 & 19.9 & 26.9 & & 22.0 \\
Microempresas & 30.9 & 27.6 & 28.1 & 29.2 & & 28.9 \\
Consumo & 35.1 & 29.8 & 30.2 & 16.5 & 37.5 & 29.8 \\
Hipotecarios & 9.3 & 9.7 & 9.2 & 10.5 & & 9.7 \\
\hline
\end{tabular}

Fuente: Superintendencia de Banco y Seguros

Elaboración: Gaby Cortez

Nota: De los bancos pequeños sólo el Banco de Comercio reporta tasas de interés 


\section{Pensamiento Crítico Vol.I7. No I}

Cuadro No. 2

Tasas de Interés Activas Anuales de las Operaciones en Moneda Extranjera de los Bancos

(Realizadas en los últimos 30 días por tipo de crédito al 29/12/2011)

\begin{tabular}{lcccccc}
\hline & \multicolumn{5}{c}{ Bancos } & \\
\cline { 2 - 6 } \multicolumn{1}{c}{ Tipo de Crédito } & Grandes & Intermedios & Medianos & Pequeños & Sucursales & Promedio \\
\hline Corporativos & 2.8 & 3.8 & 6.3 & & 4.5 & 4.3 \\
Grandes Empresas & 6.2 & 5.1 & 7.6 & 11.3 & 4.7 & 7.0 \\
Medianas Empresas & 10.2 & 7.7 & 7.7 & 14.3 & 5.2 & 9.0 \\
Pequeñas Empresas & 16.7 & 15.0 & 15.9 & 17.0 & & 16.1 \\
Microempresas & 20.7 & 14.9 & 14.9 & 17.7 & & 17.0 \\
Consumo & 22.1 & 19.8 & 15.8 & 18.3 & 25.8 & 20.3 \\
Hipotecarios & 8.1 & 8.2 & 10.4 & & 7.1 & 8.5 \\
\hline
\end{tabular}

Fuente: Superintendencia de Banco y Seguros

Elaboración: Gaby Cortez

Nota: En el caso de Bancos Pequeños la tasa que se muestra corresponde al Banco de Comercio

Se presentan algunos ejemplos para hacer más entendible la explicación que se está dando al tema. Un Banco Grande, por ejemplo, coloca en el sector corporativo el $42.62 \%$ del total de recursos destinados a dicho sector, a tasas del $5.97 \%$ en moneda nacional, y a $2.33 \%$ en moneda extranjera (tasas promedio de los últimos 30 días al 29/12/2011); y en contraposición, este mismo banco coloca el $21.68 \%$ del total de recursos dirigidos a la microempresa a tasas activas del $28.72 \%$ en moneda nacional y de $21.20 \%$ en moneda extranjera durante el mismo periodo. Evidentemente existe una diferencia ostensible en cuanto a las tasas aplicadas para cada uno de estos clientes, que es explicada por los bancos y por el riesgo que implican cada uno de estos créditos. La explicación no es motivo del presente trabajo, pero se considera que esta no puede ser la única respuesta. Lo que se puede suponer es que en los créditos con diferentes tasas de interés para un mismo servicio, el que tiene la más alta tasa de interés tendrá una probabilidad mayor de incumplimiento que el crédito otorgado con una menor tasa. En consecuencia, la situación de incumplimiento, es previsible.

\section{Tasa de interés pasiva}

Las tasas de interés pasivas, sean estas en moneda nacional o moneda extranjera, expresan la capacidad operativa de los bancos para captar recursos. Los bancos que cuentan con un número mayor de agencias a nivel nacional, y un número superior de cajeros automáticos se encuentran en condiciones más favorables para atraer depósitos. Aquí la publicidad juega un papel muy importante. 


\section{Gaby Cortez Cortez}

La magnitud de los montos captados por depósitos del público y su remuneración a través de las tasas de interés son un factor importante para la determinación del margen financiero de los bancos; así como para la tasa de retorno del capital de los bancos. A menor remuneración de los depósitos del público, en algunos casos con tasas casi inexistentes, permite a algunos bancos disponer de cantidades apreciables para su colocación en créditos con tasas de interés activas más competitivas.

Por ejemplo, los dos bancos más importantes del sistema, pagan menos del 1\% por depósitos de ahorro en moneda nacional y extranjera, pero captan más del $50 \%$ del total de obligaciones con el público. En oposición, un banco denominado pequeño paga por los depósitos de ahorro algo más de $2 \%$ en moneda nacional, pero logra captar apenas el $0.7 \%$ del total de obligaciones del público. Estos ejemplos, nos llevan a señalar las diferencias entre la dimensión de los bancos llamados grandes y la labor casi auxiliar de los bancos medianos y pequeños.

\section{Margen financiero}

El margen financiero se calcula por la diferencia entre los ratios de ingresos financieros sobre colocaciones $(\mathrm{A})$ menos el ratio de gastos financieros sobre depósitos del público (B).

El margen financiero concentra en un ratio los resultados de la actividad activa y pasiva de los bancos: por un lado, los pagos por concepto de intereses, y por otro lado, los ingresos percibidos por las colocaciones. Son una señal del grado de competencia entre los bancos o también, si se quiere desde otro punto de vista, es la forma cómo se presentan las asimetrías entre bancos como resultado de las diferentes capacidades o presencia en el mercado.

Cuando se calcula el margen para cada uno de los bancos que participan en el mercado del Perú se observa un plus en los montos del numerador debido a que se incluyen además de los intereses las comisiones. Este margen puede ser alto o bajo según como se muevan los diferentes componentes del mismo. Bajas tasas de interés en el componente activo y pasivo pueden determinar márgenes que se acerquen a un determinado benchmark. Una posicion difícil de mantener en el largo plazo por parte de los bancos pequeños es la aplicación de altas tasas activas y pasivas, debido a que 


\section{Pensamiento Crítico Vol.17. No I}

se introduce un desbalance: tasas atractivas en la parte pasiva, pero medianamente competitivas en el lado activo. Solo en nichos poco atractivos es posible que los bancos puedan desarrollar sus actividades con estos componentes. Este manejo del margen se verá reflejado en el ratio de retorno del capital (ROE) y su permanencia porcentual en el tiempo. A continuación, se muestra en el Cuadro 3 los porcentajes del margen financiero obtenidos por cada banco al 31 de diciembre de 2011:

Cuadro No. 3

\section{Margen Financiero de la Banca Múltiple}

Al 31 de diciembre de2011

(En Porcentaje)

\begin{tabular}{lccc}
\hline Bancos & (A) & (B) & $\begin{array}{c}\text { Margen } \\
\text { Financiero }\end{array}$ \\
\hline Bancos Grandes & 8.56 & 1.26 & 7.30 \\
Bancos Intermedios & 10.41 & 1.17 & 9.24 \\
Bancos Medianos & 15.59 & 2.87 & 12.72 \\
Bancos Pequeños & 41.82 & 7.01 & 34.81 \\
Bancos Sucursales & 9.13 & 1.49 & 7.75 \\
\hline
\end{tabular}

Fuente: Supertintendencia de Banca y Seguros

Elaboración : Gaby Cortez

\section{Tasa de retorno del capital (ROE)}

El ROE (Return on Equity) o Retorno del Capital es un indicador muy sencillo de calcular, pero quizás es uno de los ratios más importantes del análisis financiero. Resume las expectativas de los empresarios respecto a los ingresos que genera el capital invertido. Todo empresario busca que su capital reditúe un beneficio en las mismas condiciones que el que puede obtener cualquier otro empresario de acuerdo a su capital invertido. Esta tasa no es arbitraria ni expresada por un deseo individual, es el resultado de las fuerzas del mercado, establecida como un promedio que las empresas tratan de alcanzar según sus habilidades y productividades determinadas por el mercado en su conjunto. 


\section{Gaby Cortez Cortez}

Los capitales invertidos por los bancos difieren en sus tasas de retorno del capital que responden a la participación de los bancos en múltiples sectores económicos, diversos espacios geográficos, tipos de clientes y tasas de interés, con resultados diferentes. A continuación, se muestra el Cuadro $\mathrm{N}^{\circ} 4$ con las tasas de ROE obtenidas por cada uno de los bancos agrupados en el año 2011:

\section{Cuadro No. 4}

\section{ROE de la Banca Múltiple 2011}

(En Porcentaje)

\begin{tabular}{ll}
\hline & ROE \\
\hline Bancos Grandes & 57.16 \\
Bancos Intermedios & 40.40 \\
Bancos Medianos & 23.32 \\
Bancos Pequeños & 21.50 \\
Bancos Sucursales & 10.99 \\
\hline
\end{tabular}

Fuente: Superintendencia de Banca y Seguros

Elaboración: Gaby Cortez

Nota: En esta clasificación no incluye al Banco HSBC por tener un ROE negativo.

Actualmente el HSBS está cerrando sus operaciones en el Perú.

Si se observa los cuadros de margen y de ROE, se puede evidenciar que no necesariamente le debe corresponder a los altos márgenes financieros obtenidos por los bancos, altas tasas de retorno del capital (ROE). Por esta razón, los bancos buscan remunerar los depósitos con tasas pasivas bajas y con tasas activas que les permitan obtener un margen que se traduzca en un ROE alto, sin comprometer el desarrollo de los clientes activos de los bancos ni el riesgo por morosidad.

\section{Conclusiones}

La estructura y tipo de crédito en la banca múltiple del Perú se caracteriza por una jerarquía mediante la cual los bancos con mayor capacidad, en activos y pasivos, ocupan el mayor número de espacios económicos y áreas geográficas del mercado, prestando sus servicios a clientes que a su vez tienen un dominio importante en estos 


\section{Pensamiento Crítico Vol.17. No I}

sectores, los cuales reciben recursos a tasas de interés preferenciales con respecto a otros clientes con menor poder económico, quienes pagan más por el mismo servicio recibido. El riesgo como explicación para esta diferenciación en el tratamiento de las tasas de interés activas no parece del todo válida, por lo que amerita considerar otros elementos que también pueden explicar este comportamiento.

Los bancos a los cuales se les denomina medianos y pequeños se ubican en áreas o nichos que inicialmente no han sido atractivos para los bancos grandes e intermedios, pero que significan un espacio de negocio incentivado por una demanda creciente, $y$ que puede ser atendido por estos bancos; con tasas altas de interés por el lado pasivo para captar clientes, como por el lado activo, en sus colocaciones para obtener un margen que trate de acercarse al margen promedio del mercado. Una vez que estos nichos se vuelven atractivos, los bancos grandes ingresan a estas áreas poniendo en riesgo la permanencia o capacidad competitiva de los bancos medianos y pequeños.

El análisis de las cifras permiten concluir que no existe una correspondencia directa entre las tasas de margen financiero altas con las tasas de retorno del capital (ROE) altas. El margen y el ROE son el resultado de las estrategias que aplican los bancos, buscando tasas de interés pasivas bajas, con tasas de interés activas competitivas que pueden lograr determinados bancos que ostentan poder económico, y que ocupan el mayor número de sectores económicos, con clientes importantes en dichos sectores, los cuales cuentan con una infraestructura dentro de un amplio espacio geográfico nacional.

\section{Bibliografía}

Memorias y boletines de la superintendencia de Banca y Seguros. 